\title{
Avaliação da densificação do diboreto de titânio sinterizado por plasma pulsado
}

\section{Evaluation of spark plasma sintering on titanium diboride densification}

Luiz Antônio Fonseca Peçanha Júnior ${ }^{1}$, Ítalo do Valle Tomaz ${ }^{1}$, Ana Lúcia Diegues Skury², Michel Picanço Oliveira ${ }^{2}$

\begin{abstract}
${ }^{1}$ Laboratório de Ensaios dos Materiais - LEMat - Instituto Federal Fluminense campus Cabo Frio, Estrada Cabo Frio - Búzios, s/n, Baía Formosa, CEP: 28909-971, Cabo Frio, RJ. e-mail: luizantoniofpjunior@gmail.com, italovtomaz@gmail.com

${ }^{2}$ Laboratório de Materiais Super duros - Universidade Estadual do Norte Fluminense Darcy Ribeiro, Av. Alberto Lamego, 2000, Parque California, CEP: 28035-200, Campos dos Goitacazes - RJ. e-mail: luciaskury@hotmail.com, michelpicanco@gmail.com
\end{abstract}

\section{RESUMO}

O diboreto de titânio $\left(\mathrm{TiB}_{2}\right)$ é um material cerâmico que se destaca por sua elevada resistência mecânica, estabilidade química e dureza a altas temperaturas, entretanto, características como baixa sinterabilidade e tenacidade a fratura limitam a atuação deste material em aplicações termomecânicas. Técnicas de sinterização não convencionais como SPS e aditivo de sinterização como $\mathrm{AlN}$ e $\mathrm{Si}_{3} \mathrm{~N}_{4}$ podem reduzir a temperatura de sinterização e aumentar a tenacidade à fratura do $\mathrm{TiB}_{2}$. Neste sentido, o presente trabalho teve como objetivo avaliar a eficiência da técnica de sinterização por plasma pulsado (Spark Plasma Sintering - SPS) no processamento do diboreto de titânio. O nitreto de alumínio foi utilizado como aditivo para reduzir temperatura e tempo de sinterização e facilitar a densificação do $\mathrm{TiB}_{2}$. Os resultados apontam a sinterização por plasma pulsado como uma técnica eficiente na densificação de compósito cerâmico $\mathrm{TiB}_{2}-\mathrm{AlN}$. Elevado grau de densificação foi alcançado mesmo utilizando tempos de sinterização relativamente curto.

Palavras-chave: Diboreto de titânio, sinterização, densificação.

\begin{abstract}
Titanium diboride $\left(\mathrm{TiB}_{2}\right)$ is a ceramic material that highlight because of its high mechanical resistance, chemical stability and hardness in high temperatures. However, characteristics as low sinterability and fracture toughness restrict its usage on termomechanical applications. Non-conventional sintering techniques as SPS and sintering aid as $\mathrm{AlN}$ and $\mathrm{Si}_{3} \mathrm{~N}_{4}$ can reduce sintering temperature and increase fracture toughness of $\mathrm{TiB}_{2}$. The objective of this work is to evaluate the efficiency of spark plasma sintering (SPS) technique on the manufacturing of titanium diboride. Aluminum nitride (AIN) was used as additive to reduce sintering temperature and time and enhance $\mathrm{tib}_{2}$ densification. The results indicate that spark plasma sintering is an efficient technique in the densification of $\mathrm{TiB}_{2}-\mathrm{AlN}$ ceramic composite. High degree of densification was achieved even using relatively short sintering time.
\end{abstract}

Keywords: Titanium Diboride, Sintering, Densification.

\section{INTRODUÇÃO}

$\mathrm{O} \mathrm{TiB}_{2}$ é um composto refratário com propriedades atrativas, como elevada dureza (cerca de três vezes maior do que o aço endurecido) e resistência ao desgaste a temperaturas elevadas. A combinação de propriedades do $\mathrm{TiB}_{2}$ faz deste material um candidato a aplicações diversificadas, entre elas, material estrutural para alta temperatura e revestimentos resistentes ao desgaste [1,2]. Entretanto, a densificação do $\mathrm{TiB}_{2}$ é muito difícil e complexa, principalmente devido à elevada energia das ligações químicas entre o titânio (Ti) e o boro (B) e o seu baixo coeficiente de difusão. Assim, o transporte de massa necessário para se alcançar uma boa densificação é restrito, o que exige altas temperaturas e longos tempos de sinterização para a densificação do $\mathrm{TiB}_{2}$ utilizando técnicas convencionais de sinterização [3]. Altas temperaturas e longos tempos de sinterização 
invariavelmente geram um crescimento de grão desfavorável e degradam suas propriedades, como por exemplo dureza a quente e sua resistência mecânica $[4,5]$.

Técnicas modernas de sinterização e a utilização de materiais auxiliares vêm demonstrando sucesso na densificação de materiais com baixo coeficiente de difusão. Entre essas novas técnicas destaca-se a sinterização por plasma pulsado (Spark Plasma Sintering - SPS), também conhecido como Sinterização por centelha de plasma. Esta é uma técnica de densificação de pós caracterizada pela aplicação simultânea de pressão uniaxial e corrente contínua pulsada (on-off). O princípio da transferência de massa utilizado neste processo permite a aplicação de altas taxas de aquecimento e de resfriamento, promovendo elevada densificação com menor crescimento de grãos, mantendo a integridade das propriedades intrínsecas dos pós (dureza, resistência mecânica, estabilidade química, entre outras) nos produtos densificados [6]. Nos pós não condutores, o fluxo de calor ocorre a partir da matriz e pistões de grafite por condutividade térmica. Neste caso, a matriz e os pistões são aquecidos devido a sua própria resistência elétrica. Esta possibilidade torna a técnica de sinterização por plasma pulsado eficiente para materiais condutores e não condutores [7].

Outra solução para melhorar a sinterabilidade, e também a tenacidade à fratura do $\mathrm{TiB}_{2}$, é a utilização de um segundo composto com o objetivo de auxiliar a densificação deste material. Com o aditivo de sinterização também é possível desenvolver mecanismos de tenacificação em materiais cerâmicos. Entre os diferentes auxiliares de sinterização, o nitreto de alumínio (AIN) vem obtendo sucesso na densificação do diboreto de titânio. O AlN passou a ser estudado e desenvolvido para aplicações termomecânicas, principalmente devido à alta resistência mecânica, excelente estabilidade em altas temperaturas, baixa constante dielétrica, baixa densidade $\left(3,26{\mathrm{~g} . \mathrm{cm}^{-3}}^{-3}\right)$ e um coeficiente de expansão térmico próximo ao do silício $\left(4,8 \times 10^{6} \mathrm{~K}^{-1}\right)[8$, $9,10]$.

O presente trabalho teve como principal objetivo avaliar a eficiência da técnica de sinterização por plasma pulsado na densificação do diboreto de titânio utilizando como aditivo o nitreto de alumínio. Além disso, avaliar o efeito da temperatura máxima na sinterização do sistema $\mathrm{TiB}_{2}$ - $\mathrm{AlN}$.

\section{MATERIAIS E MÉTODOS}

Os materiais base utilizados neste trabalho foram AlN e $\mathrm{TiB}_{2}$. Ambos fornecidos por Sigma - Aldrich sob a forma de pó, com um tamanho médio de partícula de $10 \mu \mathrm{m}$. As matérias primas foram misturadas em uma proporção $70 \%$ (em peso) de $\mathrm{TiB}_{2}$ e $30 \%$ (em peso) de AlN por se tratar de composição ainda não investigada. A mistura foi realizada manualmente num almofariz com um pilão durante 30 minutos em temperatura ambiente, aproximadamente $25{ }^{\circ} \mathrm{C}$. Em seguida, as composições foram secas a $60^{\circ} \mathrm{C}$ durante 8 horas num forno eléctrico de laboratório. Os compósitos densificados foram obtidos via sinterização por plasma pulsado (SPS), utilizando equipamento "DR. SINTER LAB Jr.", modelo SPS 211 LX. A sinterização foi realizada a uma pressão constante de $80 \mathrm{MPa}$ e vácuo de $10^{-2}$ Torr para diferentes temperaturas de sinterização: $1500{ }^{\circ} \mathrm{C}$; $1600{ }^{\circ} \mathrm{C} ; 1700{ }^{\circ} \mathrm{C} ; 1800{ }^{\circ} \mathrm{C} ; 1900{ }^{\circ} \mathrm{C}$. A taxa de aquecimento foi de $200{ }^{\circ} \mathrm{C} / \mathrm{min}$, com tempo de permanência de 10 minutos na temperatura máxima. A mistura utilizada em cada procedimento de sinterização foi carregada em molde de grafite com 10,0 mm de diâmetro interno (Figura. 1).

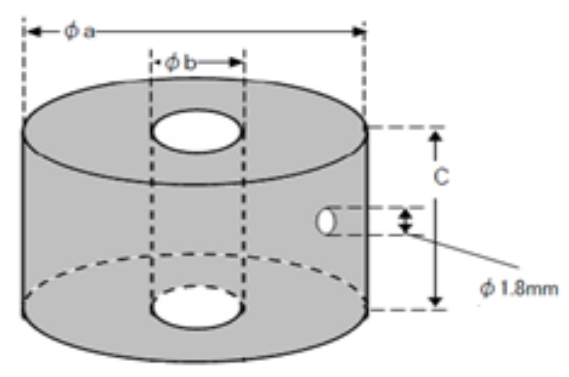

(i)

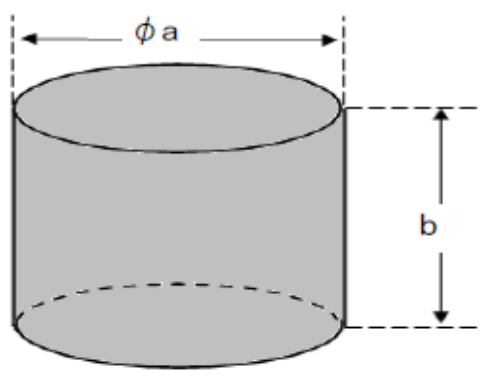

(ii)

Figura 1: (i) Matriz cilíndrica de grafite densificado: $\Phi \mathrm{a}=30,0 \mathrm{~mm} ; \Phi \mathrm{b}=10,5 \mathrm{~mm} ; \mathrm{C}=30,0 \mathrm{~mm}$; (ii) Pistões de grafite densificado, onde: $\Phi \mathrm{a}=10,0 \mathrm{~mm} ; \mathrm{b}=30,0 \mathrm{~mm}$.

O resfriamento do sistema, após sinterização, foi realizado de forma livre, aliviando a pressão uniaxial e encerrando a alimentação da corrente elétrica. Foram obtidos materiais compósitos com aproximadamente $10,0 \mathrm{~mm}$ de diâmetro, altura entre $2,82 \mathrm{~mm}$ e $3,25 \mathrm{~mm}$ e massa variando de $0,668 \mathrm{~g} \mathrm{a} \mathrm{0,698} \mathrm{g}$ (Figura 2). 

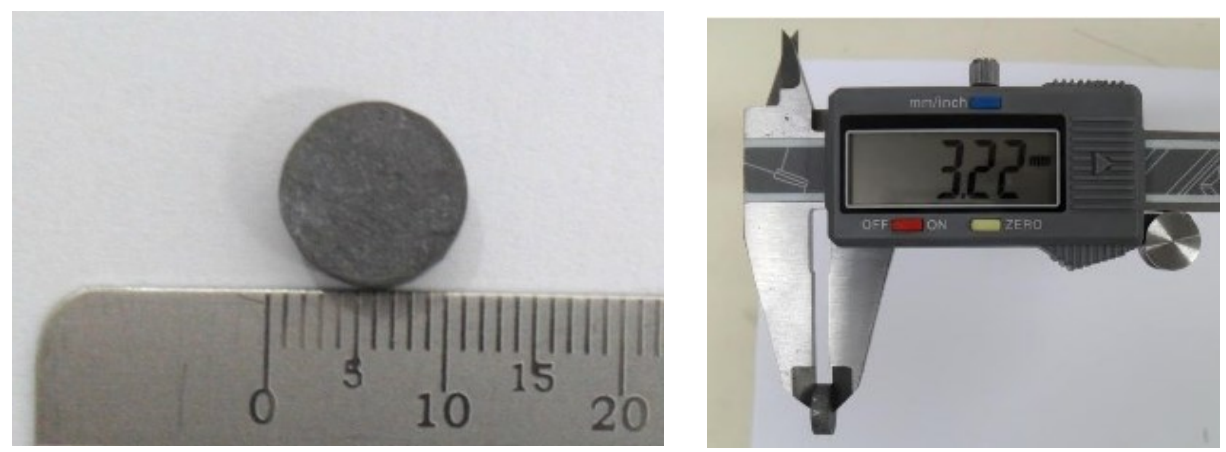

Figura 2: Imagens de uma das amostras sinterizadas.

A densidade relativa dos compósitos sinterizados foi determinada utilizando o Princípio de Arquimedes. Após a determinação da densidade relativa, as amostras foram seccionadas transversalmente e a superfície de corte foi submetida à microscopia confocal de varredura a laser (microscópio confocal da marca Olympus, modelo LEXT - 3D 4000) com a finalidade de avaliar a efetividade da sinterização através de componentes estruturais, entre eles, porosidade e segregações. Para finalizar, microanálise química foi realizada na seção de corte, com espectrômetro de energia dispersa (EDS), acoplado a um microscópio eletrônico de varredura, para determinação qualitativa e semi-qualitativa dos elementos presentes nas amostras sinterizadas.

\section{RESULTADOS}

A Figura 3 mostra as curvas de sinterização (temperatura x tempo) com o registro da temperatura e do espaçamento entre os pistões (conhecida como curva de displacement) ao longo das sinterizações em $1500{ }^{\circ} \mathrm{C}$ e $1900{ }^{\circ} \mathrm{C}$. Em termos gerais, a curva de displacement é o registro da movimentação dos pistões durante o processo. Aclives indicam aproximação entre os pistões, e consequentemente, redução de volume da amostra e sua densificação. Em contrapartida, declives da curva de displacement indicam expansão do compactado que pode ser justificado em muitos casos pela formação de novas fases ao longo do processo de sinterização.

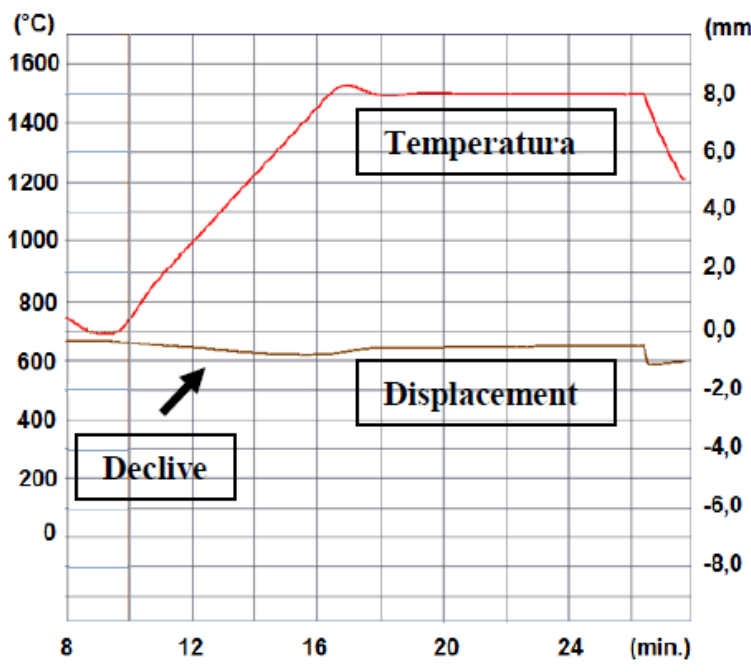

(a)

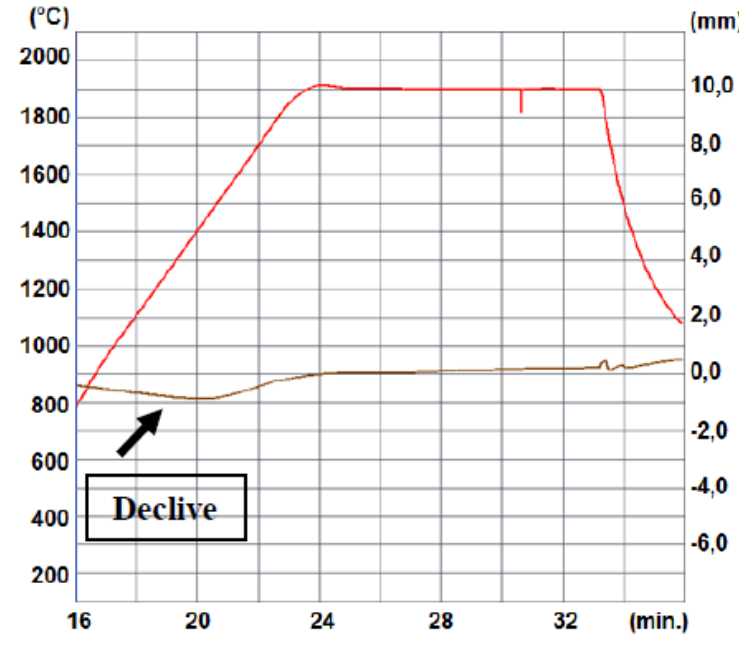

(b)

Figura 3: Curvas de temperatura e de displacement ao longo da sinterização: (a) $1500{ }^{\circ} \mathrm{C}$; (b) $1900{ }^{\circ} \mathrm{C}$.

A Figura 4 mostra o gráfico da densidade relativa em função da temperatura para as amostras sinterizadas. As amostras sinterizadas em $1900{ }^{\circ} \mathrm{C}$ atingiram densidade relativa de $93,5 \%$, aquelas sinterizadas em $1800{ }^{\circ} \mathrm{C}$ alcançaram densidade de $92,7 \%$. Já as amostras sinterizadas abaixo de $1800{ }^{\circ} \mathrm{C}$ não alcançaram densidade relativa acima de $90 \%$. A densidade de amostras sinterizadas em temperaturas de $1700{ }^{\circ} \mathrm{C}$ atingi$\operatorname{ram} 87,5 \%, 1600{ }^{\circ} \mathrm{C}$ e $1500{ }^{\circ} \mathrm{C}$ densificaram respectivamente $82,4 \%$ e $75 \%$ do seu valor teórico. 


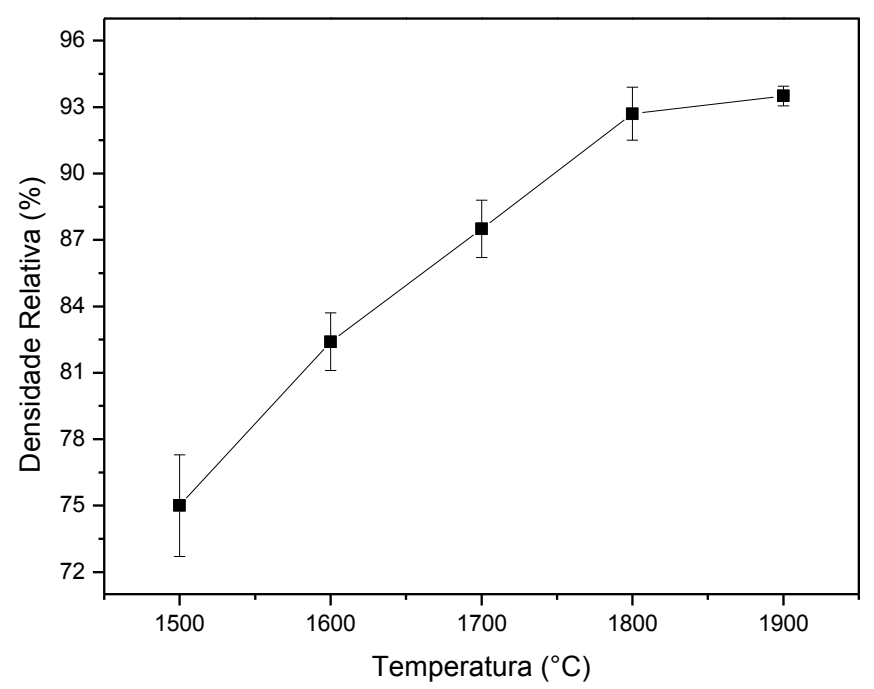

Figura 4: Densidade relativa das amostras em função da temperatura de sinterização.

Imagens topográficas tridimensionais obtidas por microscopia confocal são apresentadas na Figura 5. Destaque para a grande irregularidade na superfície da amostra sinterizada em $1500{ }^{\circ} \mathrm{C}$ (Figura 5.a), quando comparada às amostras sinterizadas em temperaturas superiores. Fato que se justifica pela menor porosidade das amostras sinterizadas em temperaturas maiores. Este tipo de análise é adequado para avaliar a efetividade da sinterização através de componentes estruturais tais como, a porosidade. Análise possível principalmente devido a facilidade da microscopia confocal para aumentar o contraste em uma imagem microscópica. As seções ópticas podem fornecer uma construção óptica em 2D (2 dimensões) e 3D (3 dimensões). Uma vez que, no microscópio Confocal todas as estruturas fora de foco são eliminadas é possível obter melhor definição e profundidade de campo em relação à microscopia óptica convencional. Entretanto, a preparação das amostras pode influenciar nos resultados obtidos. Neste sentido, em alguns casos amostras com menor densidade relativa pode apresentar superfícies mais lisas quando comparadas a amostras de maior densidade.

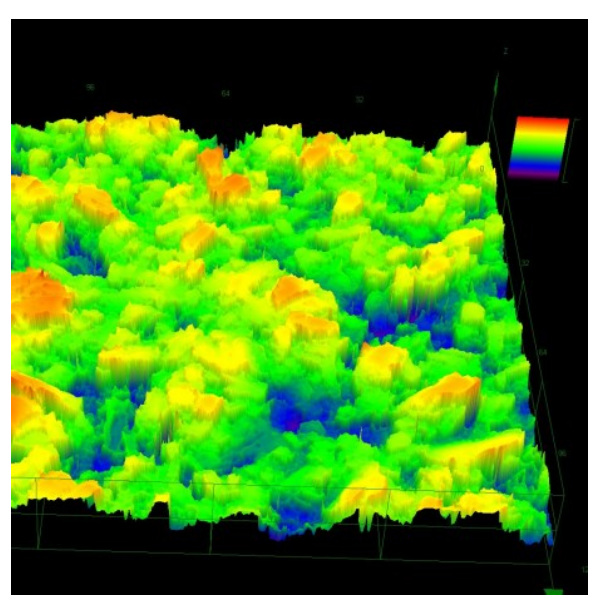

(a)

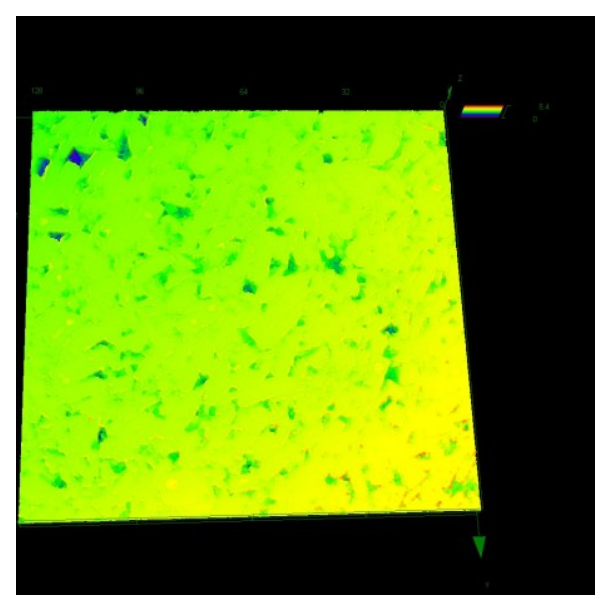

(b) 


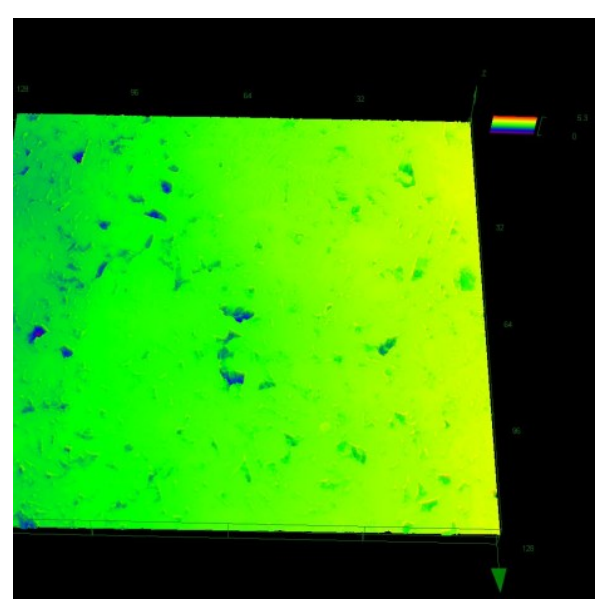

(c)

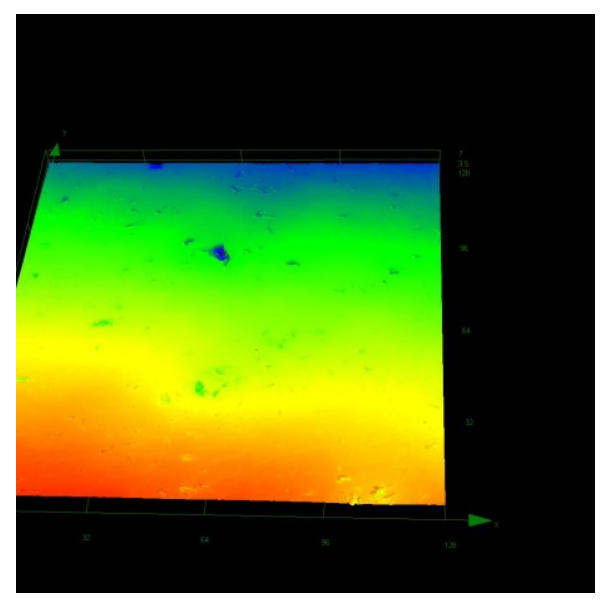

(e)

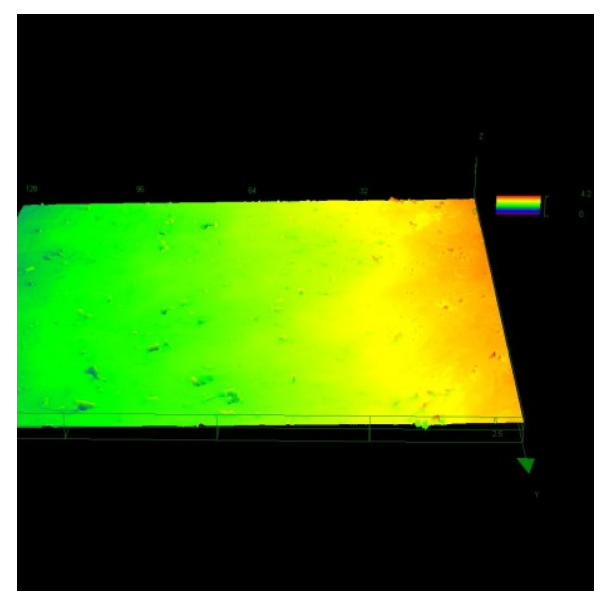

(d)

Figura 5: Imagem topográfica tridimensional obtida por microscopia confocal para as amostras sinterizadas em diferentes temperaturas, onde: (a) $1500{ }^{\circ} \mathrm{C}$; (b) $1600{ }^{\circ} \mathrm{C}$; (c) $1700{ }^{\circ} \mathrm{C}$; (d) $1800{ }^{\circ} \mathrm{C}$; (e) $1900{ }^{\circ} \mathrm{C}$. Aumento de $2136 \times$ (escala de profundidade: mícrons).

A figura 6 apresenta a microanálise química por EDS realizada em amostra sinterizada em $1500{ }^{\circ} \mathrm{C}$ e $1900{ }^{\circ} \mathrm{C}$. O mapeamento foi realizado destacando as regiões com alta concentração de alumínio e titânio na tentativa de identificar o nitreto de alumínio e o diboreto de titânio após o procedimento de sinterização. As imagens obtidas por microscopia eletrônica de varredura permitiram avaliar a homogeneidade da sinterização dos cristais de $\mathrm{TiB}_{2}-\mathrm{AlN}$ e estabelecer uma relação entre a temperatura de sinterização e os fenômenos de densificação alcançados. 


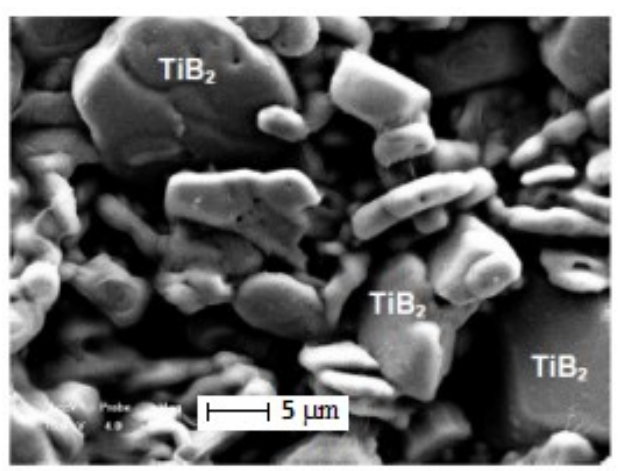

(a)
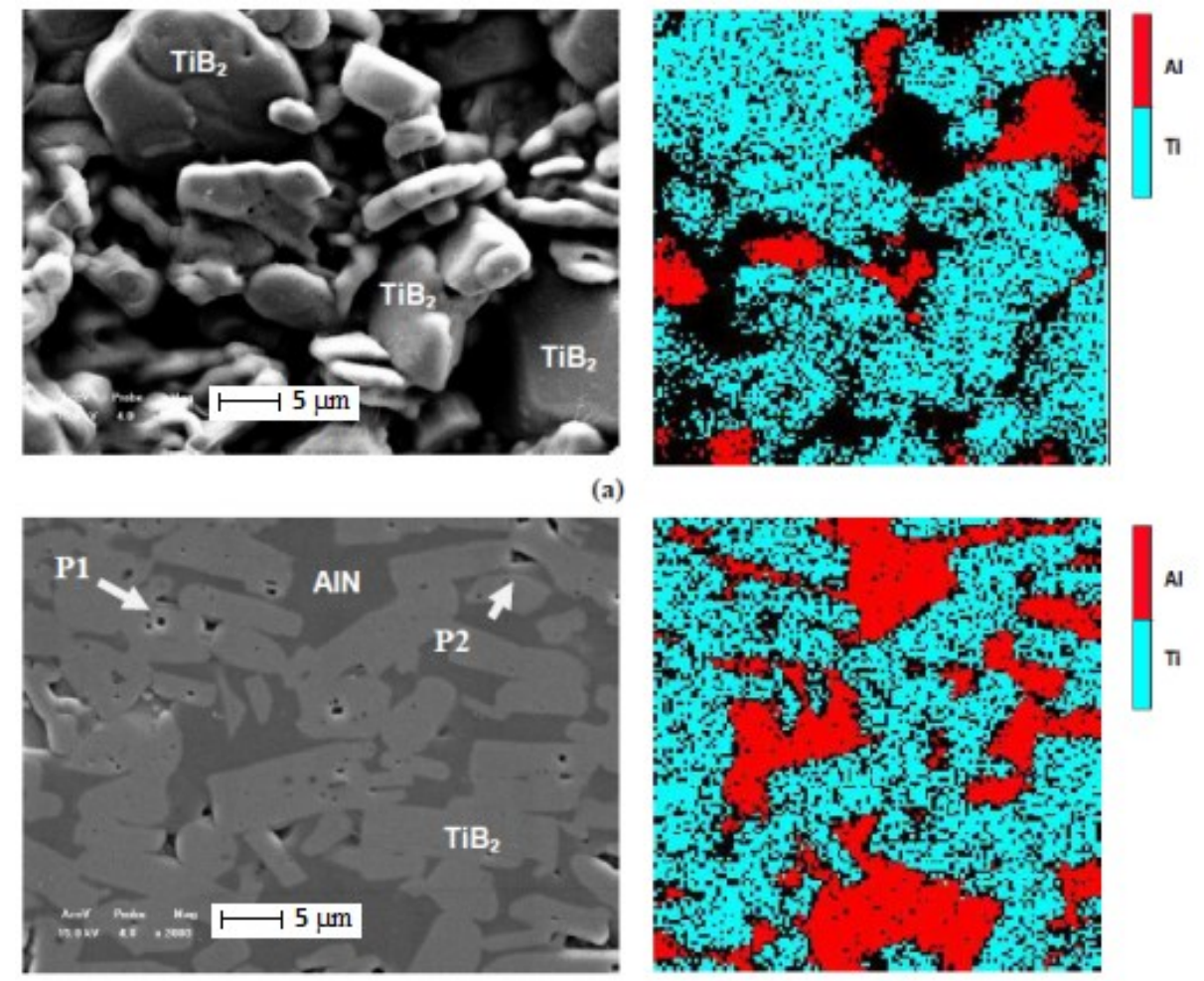

(b)

Figura 6: Mapeamento por EDS para amostra sinterizada: (a) $1500{ }^{\circ} \mathrm{C}$; (b) $1900{ }^{\circ} \mathrm{C}$. $\mathrm{P} 1=$ poros esféricos no centro dos grãos de $\mathrm{TiB}_{2} ; \mathrm{P} 2$ = poros agudos nas interfaces do sinterizado. Microscópio Eletrônico de Varredura com aumento de $2000 \mathrm{x}$.

\section{DISCUSSÃO}

As curvas de temperatura e deslocamento são ferramentas importantes para acompanhar e registrar o comportamento da mistura e a variação de temperatura do sistema ao longo do processo de sinterização. $\mathrm{O}$ menor aclive da curva de displacement em amostras sinterizadas em $1500^{\circ} \mathrm{C}$ (Figura 3.a) foi o primeiro indicador da baixa densificação alcançada a esta temperatura. Já as amostras sinterizadas em $1900{ }^{\circ} \mathrm{C}$ (Figura 3.b) registraram maior aclive na curva de displacement, apontando para maior grau de densificação do sistema. Neste sentido, as curvas de sinterização confirmam a baixa sinterabilidade do diboreto de titânio.

Analisando o gráfico de densidade relativa na figura 4 é possível observar que as amostras corresponderam às expectativas e aumentaram o grau de densificação com o aumento da temperatura de sinterização. Mesmo com a técnica SPS e a utilização do nitreto de alumínio como auxiliar de sinterização não foi possível alcançar densidades relativas satisfatórias para temperaturas abaixo de $1800{ }^{\circ} \mathrm{C}$. Entretanto, as amostras atingiram densidades relativas acima de $90 \%$, quando sinterizadas a $1800{ }^{\circ} \mathrm{C}$ e $1900{ }^{\circ} \mathrm{C}$ com tempo de sinterização (10 minutos) curto quando comparado a técnica convencional de sinterização. Temperaturas acima de $1900{ }^{\circ} \mathrm{C}$ não foram avaliadas devido à limitação do modelo de equipamento utilizado. As temperaturas de $1800{ }^{\circ} \mathrm{C}$ e $1900{ }^{\circ} \mathrm{C}$ sem dúvida foram as temperaturas que demonstraram melhores resultados, entretanto para determinar a temperatura ideal de sinterização deste sistema será necessário a avaliação de propriedades, como dureza e tenacidade a fratura. A eficiência da técnica de sinterização utilizada fica evidente quando comparada a técnicas convencionais que podem levar horas para obter a densificação do diboreto de titânio utilizando as mesmas temperaturas e auxiliares de sinterização. Um exemplo é a técnica de prensagem a quente, que utilizando o mesmo AlN como aditivo na sinterização do $\mathrm{TiB}_{2}$, necessita de aproximadamente 1 hora para alcançar densificação acima de $90 \%$ em $1800{ }^{\circ} \mathrm{C}$ [11].

A análise microestrutural dos compósitos sinterizados permitiu avaliar os mecanismos e fenômenos de sinterização ocorridos nas diferentes temperaturas utilizadas. Com as imagens topográficas tridimensionais obtidas por microscopia confocal (Figura 5) é possível observar o fechamento de poros e a densificação do sistema com o aumento da temperatura de sinterização. As amostras sinterizadas em $1500{ }^{\circ} \mathrm{C}$ (Figura 5.a) registram grande irregularidade topográfica, resultado direto de sua baixa densificação. Ainda analisando as 
imagens obtidas por microscopia confocal é possível observar que o aumento da temperatura de sinterização resulta em redução gradual das irregularidades topográficas nas amostras sinterizadas. Tal comportamento é justificado pela redução da porosidade com o aumento da temperatura de sinterização.

A microscopia confocal não foi suficiente para avaliar as particularidades microestruturais dos compósitos sinterizados, como por exemplo, a morfologia dos poros ainda presentes. Com o objetivo de aprofundar esta investigação, a microanálise química por EDS acoplada a um microscópio eletrônico de varredura (Figura 6) foi utilizada. Os resultados obtidos com a análise realizada em amostras sinterizadas em $1500{ }^{\circ} \mathrm{C}$ (Figura 6.a) confirmam a dificuldade de sinterizar $\mathrm{TiB}_{2}$ a temperaturas relativamente baixas. É possível observar na amostra sinterizada em $1500{ }^{\circ} \mathrm{C}$ a presença de particulas de $\mathrm{TiB}_{2}$ com morfologia muito próxima a sua forma original, denotando que sofreram pouca sinterização. Tal fenômeno se justifica por um baixo coeficiente de difusão do diboreto de titânio, necessitando de maior energia de ativação para atingir os estágios e fenômenos de sinterização (difusão por volume e contorno de grão) responsáveis pela densificação do sistema $[12,13]$.

A microanálise química por EDS realizada na amostra sinterizada em $1900{ }^{\circ} \mathrm{C}$ (Figura 6.b) foi importante para a identificação de uma matriz de AlN (30\% em peso do compósito) e fase dispersa de $\mathrm{TiB}_{2}(70 \%$ em peso do compósito) na estrutura dos sinterizados. Além disso, é possível observar a morfologia original do pó de partida do diboreto de titânio. Essa observação é de grande relevância, uma vez que, o formato alongado dos grãos de $\mathrm{TiB}_{2}$, associada à diferença de coeficiente de expansão térmica existente entre este composto e o AlN, são responsáveis por mecanismos de tenacificação comuns em compósitos cerâmicos. Entre esses mecanismos destaca-se a deflexão de trincas e a propagação de trincas através dos contornos de grão. A deflexão se caracteriza pela mudança sistemática da direção de propagação da trinca, fazendo com que a tensão aplicada deixe de ser normal à superfície da trinca, reduzindo as tensões na frente de propagação. Com isso, será necessária maior energia para que a trinca alcance tamanho crítico e se torne catastrófica. Já a propagação intergranular ocorre devido a contornos de grãos suficientemente fracos, favorecendo a propagação de trincas através dos contornos de grão, o que aumenta a tenacidade principalmente, se a microestrutura apresentar grãos alongados [5,14]. A confirmação destes mecanismos de tenacificação só será possível com a avaliação das propriedades mecânicas dos sinterizados.

Ainda analisando a Figura 6 é possível observar a coalescência entre os grãos de $\mathrm{TiB}_{2}$ e poros com diferentes geometrias: poros esféricos no centro dos grãos de $\mathrm{TiB}_{2}(\mathrm{P} 1)$ e poros agudos nas interfaces do sinterizado (P2). Poros com formato esférico no centro dos grãos de $\mathrm{TiB}_{2}(\mathrm{P} 1)$ são aqueles resultantes da sinterização direta de partículas de diboreto de titânio que não chegou a eliminar completamente a porosidade. Entretanto, poros com arestas agudas (P2) são resultado da sinterização entre o $\mathrm{TiB}_{2}$ e o AlN, a presença destes poros provavelmente é o reflexo da dificuldade do nitreto de alumínio para molhar a superfície dos grão de diboreto de titânio em algumas regiões [12].

\section{CONCLUSÕES}

- A técnica de sinterização por plasma pulsado demonstrou sucesso na sinterização do sistema $\mathrm{TiB}_{2}$-AlN. Foram alcançada altas densificações em curto intervalo de tempo, principalmente quando comparado a técnicas convencionais utilizadas na sinterização deste mesmo sistema.

- Os compósitos cerâmicos $\mathrm{TiB}_{2}$ - AlN alcançaram densidade relativa acima de $90 \%$ quando sinterizados em $1900{ }^{\circ} \mathrm{C}$.

- Os compósitos que obtiveram altas densidades relativas apresentaram uma matriz de AlN envolvendo a fase dispersa de $\mathrm{TiB}_{2}$.

- Mesmo a temperaturas elevadas de sinterização foi possível observar a presença de poros, com diferentes mofologias, que afirmam a baixa sinterabilidade do $\mathrm{TiB}_{2}$.

- Apesar da efíciência da técnica de sinterização por plasma pulsado não foi possível obter compósitos com densidade relativa acima de $90 \%$ utilizando temperaturas de sinterização inferior a $1800{ }^{\circ} \mathrm{C}$.

\section{AGRADECIMENTOS}

Os autores agradecem o apoio a esta investigação por parte das agências brasileiras: CNPq, CAPES e FAPERJ . 


\section{BIBLIOGRAFIA}

[1] BASU, B., RAJU, G. B., SURI, A. K., "Processing and properties of monolithic $\mathrm{TiB}_{2}$ based materials", International Materials Reviews, v. 51, n. 6, pp. 352-374, 2006.

[2] ZHANG Z.H., SHEN X.B., WANG F.C., et al., "Densification behavior and mechanical properties of the spark plasma sintered monolithic TiB2 ceramics", Materials Science and Engineering, v.527, pp.5947-51, 2010.

[3] MUNIR, Z. A., ANSELMI-TAMBURINI, U., OHYANAGI, M., "The effect of electric field and pressure on the synthesis and consolidation of materials: a review of the spark plasma method", Journal of Materials Science, v.41, pp. 763-777, 2006.

[4] WANG, W., FU, Z., WANG, H., et al., "Influence of hot pressing temperature and time on microstructure and mechanical properties of $\mathrm{TiB}_{2}$ ceramics", Journal of the European Ceramic Society, v.22, pp.1045-9, 2002.

[5] SHENG, C., JASON, G. S., DEV, B., "Titanium diboride composite with improved sintering characteristics", Int. Journal of Refractory Metals and Hard Materials, v.49, pp. 314-319, 2015.

[6] ZIEMNICKA-SYLWESTER, M., "TiB 2 -based composites for ultra-high-temperature devices, fabricated by SHS, combining strong and weak exothermic reactions", Materials, v.6, pp.1903-19, 2013.

[7] RAGULYA, A.V., "Fundamentals of Spark Plasma Sintering”, Encyclopedia of Materials: Science and Technology, Elsevier Ltd., pp. 1-5, 2010.

[8] GENG-FU, XU, YUVAL, C., TAYO, O., et al., "Microwave sintering and properties of AlN/TiB ${ }_{2}$ composites", Journal Materials Research, v. 81, n. 1, pp. 66-76, 2003.

[9] BALDACIM,S. A., SILVA, O.M.M., SANTOS, C., et al., "Estudo do nitreto de alumínio para aplicações termo-mecânicas”, Revista Cerâmica, v.51, no.320, pp.349-353, Out./Dez, São Paulo, 2005.

[10] XIANG-YU, Z., SHOU-HONG, T., DONG-LIANG, J. “AIN-TiB 2 composites fabricated by spark plasma sintering”, Ceramics International, v. 31, pp 267-270, 2005.

[11] LONG-HAO, L., HYOUN-EE, K., EUL, S. K. "Sintering and mechanical properties of titanium diboride with aluminum nitride as a sintering aid", Journal of the European Ceramic Society, v.22, pp. 973-977, 2002.

[12] SONG, X., LIU, X., ZHAN G,J., "Neck formation and self-adjusting mechanism of neck growth of conducting powders in spark plasma sintering", Journal of the American Ceramic Society, v.89, pp. 494-500, 2006.

[13] ZHANG, Z.H., SHEN, X.B., WANG, F.C., et al., "Low-temperature densification of $\mathrm{TiB}_{2}$ ceramic by the spark plasma sintering process with Ti as a sintering aid”, Scripta Materialia, v.66, pp.167-170, 2012.

[14] KRUZIC, J.J., SATET, R.L., HOFFMANN, M.J., et al., "The utility of R-curves for understanding fracture toughness-strength relations in bridging ceramics", Journal of the American Ceramic Society, v. 9, n. 6, pp. 1986-1994, 2008. 